\title{
Cyclo[18]carbon: smallest all-carbon electron acceptor
}

\author{
Anton J. Stasyuk, ${ }^{a^{*}}$ Olga A. Stasyuk, ${ }^{a}$ Miquel Sola, ${ }^{a^{*}}$ and Alexander A. Voityuk ${ }^{a, b^{*}}$
}

Received 00th January 20xx

Accepted 00th January 20xx

DOI: $10.1039 / \times 0 \times x 00000 x$

Recently synthesized $C_{18}$ molecule demonstrates strong electron acceptor properties similar to the $\mathrm{C}_{60}$. In this work, we study computationally the ground and excited state properties of $\mathrm{C}_{18}$ and its complexes with several electron-donating molecules. We demonstrate that a high amount of the exact (HF) exchange in the DFT functional leads to a polyynic structure of $C_{18}$, in agreement with experiment. We show that in complexes of $C_{18}$ with electron donors, the lowest excited states are charge separated states formed by electron transfer to $C_{18}$. This makes $C_{18}$ to be the smallest all-carbon electron acceptor reported so far. Because $C_{18}$ exhibits a larger internal reorganization energy as compared to fullerene $C_{60}$, ET reactions with relatively high driving force will be shifted from the inverted to the normal Marcus regime when replacing $C_{60}$ by $\mathrm{C}_{18}$.

Since the first generation of cyclo[18]carbon in 1989, small carbon clusters have attracted much attention of both experimentalists and theoreticians. ${ }^{1}$ In contrast to threecoordinated carbon atoms in fullerenes, carbon nanotubes and graphene, the $\mathrm{C}_{18}$ allotrope possesses only two-coordinated carbon atoms. The existence of $\mathrm{C}_{18}$ in the gas-phase as a highly reactive species has been repeatedly demonstrated. ${ }^{2}$ The reactivity of $C_{18}$ prevented the experimental determination of its geometry and encouraged numerous quantum-mechanical calculation of its structure. ${ }^{3}$ The cyclic carbon molecules have two $\pi$-conjugated electronic systems described by out-of-plane and in-plane $\pi$-molecular orbitals (MOs) that provide different contributions to their stability. Two symmetric geometries have been considered for $C_{18}$ : a non-aromatic $D_{9 h}$ polyyne structure with alternating single and triple bonds and a doubly Hückel aromatic cumulenic $\left(D_{18 h}\right)$ structure having only $C=C$ double bonds. ${ }^{4}$ Note that most DFT calculations of $C_{18}$ predict the $D_{18 \mathrm{~h}}$ symmetry $1 \mathrm{c}, 3 \mathrm{~b}, 5$ whereas the Hartree-Fock (HF) and Coupled Cluster methods give the $D_{9 h}$ structure, ${ }^{3 d, 5,6}$ the $D_{18 h}$ structure

\footnotetext{
a) Dr. A. J. Stasyuk, Dr. O. A. Stasyuk, Prof. Dr. M. Solà, Prof. Dr. A. A. Voityuk Institute of Computational Chemistry and Catalysis and Department of Chemistry University of Girona

C/M. Aurèlia Capmany, 69, 17003 Girona, Spain.

E-mail: antony.stasuk@gmail.com

E-mail:miquel.sola@udg.edu

E-mail: alexander.voityuk@icrea.cat

b)Prof. Dr. A. A. Voityuk

Institució Catalana de Recerca i Estudis Avancats (ICREA), 08010 Barcelona

Spain.

+ Footnotes relating to the title and/or authors should appear here.

Electronic Supplementary Information (ESI) available: full computational details, supporting figures and tables. See DOI: 10.1039/x0xx00000x
}

being a transition state between two $D_{9 h}$ species. ${ }^{7}$ On the basis of these data, one may suggest that the increasing amount of the exact HF exchange in a DFT functional will stabilize the polyynic structure comparing to the cumulenic one.

Recently, Kaiser et al characterized the cyclo[18]carbon by highresolution atomic force microscopy (AFM). ${ }^{8}$ The AFM study, supported by computational simulations, established the polyynic structure of $\mathrm{C}_{18}$ on the $\mathrm{NaCl}$ surface. They also demonstrated the ability of cyclo[18]carbon to exist in two charge forms - neutral (planar, symmetric) and negatively charged (less symmetric and less planar) that can be reversibly switched. DFT calculations performed by the authors using two different functionals (PBE and HSE with $25 \%$ and $80 \%$ of the exact exchange, respectively) led to contradicting results. As expected, the PBE calculation predicts the cumulenic $D_{18 \mathrm{~h}}$ structure whereas the HSE suggests the polyynic $D_{9 h}$ structure. A low LUMO energy of $\mathrm{C}_{18}$ comparable to that of $\mathrm{C}_{60}(-2.24$ and $-2.58 \mathrm{eV}$ found by CAM-B3LYP for $\mathrm{C}_{18}$ and $\mathrm{C}_{60}$ ) ensures the stability of its anion radical and makes $C_{18}$ to be an electron acceptor. One of the key characteristics that control charge mobility in organic electronics is the reorganization energy which is usually divided into two terms, internal and external (environmental) contributions. The contribution of electron acceptor to the internal reorganization energy can be estimated as the difference of the adiabatic and vertical electron affinities. Comparing DFT estimates for $\mathrm{C}_{18}$ and $\mathrm{C}_{60}$ obtained by using two closely related functionals - B3LYP and CAM-B3LYP, we have found a remarkable difference between the results (Table 1 ). The reorganization energy $\left(\lambda_{i}\right)$ of $C_{60}$ is almost independent of the functional used whereas the value of $\lambda_{i}$ of $C_{18}$ increases by a factor of 1.5 by passing from B3LYP to CAM-B3LYP. To find out which of the functionals gives more reliable estimates for $C_{18}$ we performed ab initio DLPNO-CCSD(T) computations. The highlevel calculation provides a support for the CAM-B3LYP results (Table 1).

Table 1. Computed vertical and adiabatic electron affinities (VEA and AEA) and internal reorganization $\left(\lambda_{i}\right)$ energies $C_{18}$ and fullerene $C_{60}$. All energies in $\mathrm{eV}$.

\begin{tabular}{lcccccc}
\hline \multirow{2}{*}{$\begin{array}{l}\text { FC } \\
\text { Functional }\end{array}$} & \multicolumn{3}{c}{$\mathrm{C}_{60}$} & \multicolumn{3}{c}{$\mathrm{C}_{18}$} \\
\cline { 2 - 7 } BEA & $\mathrm{AEA}$ & $\lambda_{\mathrm{i}}$ & VEA & $\mathrm{AEA}$ & $\lambda_{\mathrm{i}}$ \\
\hline BSYP & 2.39 & 2.46 & 0.07 & 2.12 & 2.45 & 0.33 \\
CAM-B3LYP & 2.16 & 2.25 & 0.09 & 1.83 & 2.37 & 0.54 \\
$\begin{array}{l}\text { DLPNO- } \\
\text { CCSD }(\mathrm{T})^{\mathrm{a}}\end{array}$ & & $\mathrm{n} / \mathrm{a}$ & & 1.24 & 1.74 & 0.50 \\
\hline
\end{tabular}

a DLPNO-CCSD(T)/Def2-TZVP//CCSD/Def2-SVP 
Obviously, the internal reorganization energy of an electron acceptor is determined by changes in its geometry associated with attachment of an electron. To characterize the structure of $\mathrm{C}_{18}$ we will use the bond length alternation (BLA) index defined as the average of adjacent bond length differences:

$$
B L A=\frac{1}{n} \sum_{i=1, n}\left|R_{i}-R_{i}^{\prime}\right|
$$

where two bonds belong to the same atom $i$. Considering the structure of the $\mathrm{C}_{18}$ anion-radical obtained with B3LYP and CAM-B3LYP we found the bond length alternation (BLA) index values to be very close to each other and equal to 0.027 and 0.039 . Thus the deviation in reorganization energies calculated with B3LYP and CAM-B3LYP should be caused by different ground state geometries of neutral $\mathrm{C}_{18}$. Indeed, the BLA index for the $\mathrm{C}_{18}$ structures $(0.000$ and $0.122 \AA$ for B3LYP and CAMB3LYP) shows that both structures are quite different). Note that the $a b$ initio couple cluster calculation predict polyynic ground state structure with BLA $=0.147 \AA$.

Having on hand the experimental structure and the data of various DFT calculations we focus our attention on a more general question: is the DFT approach capable to produce reliable results for cyclic carbon molecules, and if so then what conditions should be met? The majority of DFT calculations of $\mathrm{C}_{18}$ have been performed with popular GGA (e.g. BLYP) and hybrid (e.g. B3LYP and PBEO) functionals. Usually these functionals provide reliable results. ${ }^{9}$ However, because of a relatively small amount of exact exchange (20\% for B3LYP and $25 \%$ for PBEO) they predict the cumulenic structure for ground state of $\mathrm{C}_{18}$. In contrast, the HSE functional, with $80 \%$ of exact exchange, predicts the polyynic structure. ${ }^{8}$ Thus the symmetry ( $D_{9 h}$ or $D_{18 h}$ ) of the $C_{18}$ molecule given by DFT is determined by the weight of exact exchange in the hybrid functional. To test this assumption, we performed a series of B3LYP calculations where the amount of $\mathrm{HF}$ exchange ${ }^{10}$ was varied from $5 \%$ to $100 \%$ and found the bond length alternation (BLA) index for each optimized structure (Figure 1). Each optimization was started from both cumulenic and polyynic initial geometries. The frequencies analysis was performed for all stationary points, to confirm the minimum. Independently of the functional used the geometry always converges only to one minimum, either to polyynic $\left(D_{9 h}\right)$ or to cumulenic $\left(D_{18 h}\right)$ one. The BLA index was applied to assign each structure to the cumulenic or polyynic type. The dependence of the $C_{18}$ structure on the amount of exact exchange is shown in Figure 1. The plot is divided into 3 zones: (i) pure cumulenic structures (BLA < 0.01); (ii) polyyinic structures with BLA > 0.10 and (iii) intermediate structures with BLA in the range of 0.01-0.10. As expected, the increasing amount of exact exchange in the B3LYP functional stabilizes the polyynic structure with respect to the cumulenic one. An increase in the amount of HF exchange in the functional lead to the increase in HOMO-LUMO gap (Figure S1). Inspired by our findings, we decided to verify this dependence not only on artificially modified B3LYP functional but also for regular well-proven functionals of different type (GGA, metaGGA, hybrid and range-separated (RS) hybrid) with different amount of HF exchange (Table 2). ${ }^{11}$ The data presented in Table
2 confirm that the amount of HF exchange in the DFT functional determines the symmetry of the $\mathrm{C}_{18}$ geometry.
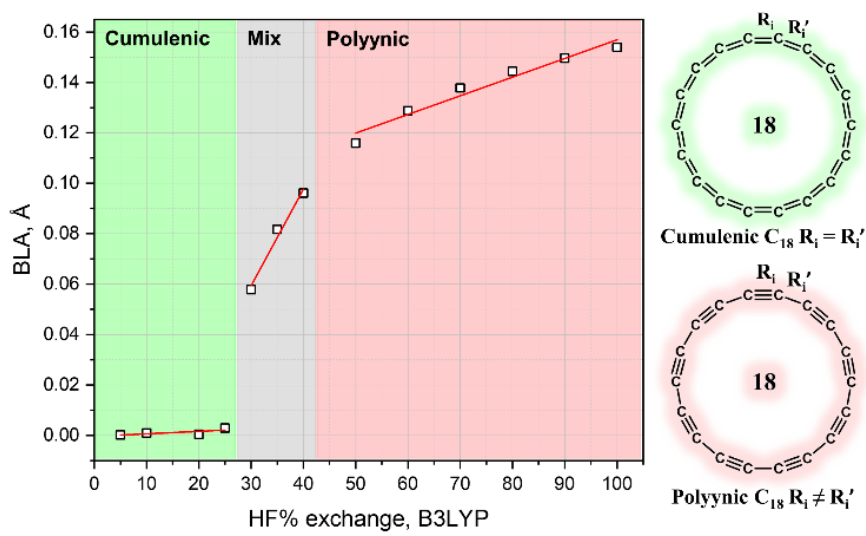

Figure 1. Dependence of alternation of the bond length in cyclo[18]carbon on the amount of exact exchange in B3LYP functional.

A coupled cluster (CCSD) calculation performed by us and reported earlier ${ }^{6}$ predicts a polyynic structure of $C_{18}$ with bond lengths of 1.238 and $1.383 \AA$. These reference values are well reproduced by the M11 and M06-HF DFT methods where the functionals have a high amount of exact exchange. Thus in contrast to doubts expressed previously, ${ }^{5,6}$ we conclude that DFT calculations give reliable results for the cyclo[18]carbon structure provided a high weight of exact exchange in the functional. In the case of cyclic carbon clusters, the DFT approach fells victim of the deficiency of its most popular functionals, all of them contain rather small amount of the exact exchange that results in a too much delocalized picture of the exchange-correlation hole. ${ }^{12}$

Table 2. Comparison of the cyclo[18]carbon lowest energy structures BLA index obtained with different $\mathrm{XC}$ functionals for singlet and triplet electronic states. All structures were optimized at XC/Def2-TZVP-D3(zero-damping) level. The cumulenic and polyynic structures are highlighted with green and red respectively, mixed structures with grey.

\begin{tabular}{|c|c|c|c|c|c|}
\hline \multirow[b]{2}{*}{$\begin{array}{l}X C \\
\text { Functional }\end{array}$} & \multirow{2}{*}{$\begin{array}{l}\mathrm{HF} \\
\text { exchange }\end{array}$} & \multicolumn{2}{|c|}{ Singlet state } & \multicolumn{2}{|c|}{ Triplet state } \\
\hline & & $\mathrm{BLA}, \AA$ & $\begin{array}{l}\text { Bond length, } \AA \\
\mathrm{R}_{\mathrm{i}} ; \mathrm{R}_{\mathrm{i}}^{\prime}\end{array}$ & $\begin{array}{l}\text { Erel, } \\
\mathrm{eV}\end{array}$ & BLA, $\AA$ \\
\hline BLYP & 0 & 0.000 & $\mathrm{R}_{\mathrm{i}}=\mathrm{R}_{\mathrm{i}}^{\prime}=1.286$ & 1.73 & 0.035 \\
\hline M06L & 0 & 0.000 & $\mathrm{R}_{\mathrm{i}}=\mathrm{R}_{\mathrm{i}}{ }^{\prime}=1.275$ & 1.75 & 0.036 \\
\hline M11L & 0 & 0.000 & $\mathrm{R}_{\mathrm{i}}=\mathrm{R}_{\mathrm{i}}^{\prime}=1.265$ & 1.74 & 0.009 \\
\hline TPSSh & 10 & 0.001 & $\mathrm{R}_{\mathrm{i}}=\mathrm{R}_{\mathrm{i}}{ }^{\prime}=1.279$ & 1.64 & 0.039 \\
\hline B3LYP & 20 & 0.000 & $\mathrm{R}_{\mathrm{i}}=\mathrm{R}_{\mathrm{i}}{ }^{\prime}=1.277$ & 1.71 & 0.046 \\
\hline PBEO & 25 & 0.000 & $\mathrm{R}_{\mathrm{i}}=\mathrm{R}_{\mathrm{i}}{ }^{\prime}=1.276$ & 1.68 & 0.046 \\
\hline M06 & 27 & 0.053 & $R_{i}=1.250 ; R_{i}^{\prime}=1.302$ & 1.60 & 0.041 \\
\hline HISSbPBE & $0-60-0^{b}$ & 0.076 & $R_{i}=1.235 ; R_{i}^{\prime}=1.310$ & 1.68 & 0.043 \\
\hline M06-2X & 54 & 0.122 & $R_{i}=1.223 ; R_{i}^{\prime}=1.345$ & 2.10 & 0.052 \\
\hline CAMB3LYP & $19-100^{b}$ & 0.122 & $R_{i}=1.219 ; R_{i}^{\prime}=1.341$ & 1.67 & 0.059 \\
\hline wB97XDa & $22-100^{b}$ & 0.125 & $R_{i}=1.220 ; R_{i}^{\prime}=1.345$ & 1.81 & 0.050 \\
\hline $\mathrm{M} 11^{\mathrm{a}}$ & $42-100^{b}$ & 0.143 & $R_{i}=1.215 ; R_{i}^{\prime}=1.358$ & 2.14 & 0.073 \\
\hline M06-HF & 100 & 0.163 & $\mathrm{R}_{\mathrm{i}}=1.207 ; \mathrm{R}_{\mathrm{i}}^{\prime}=1.371$ & 2.58 & 0.100 \\
\hline
\end{tabular}

a Empirical dispersion efficiently included in the functional; $b$ Percentage of $\mathrm{HF}$ exchange in the short, middle (only for HISSBPBE) and long range. 
The ground state of $\mathrm{C}_{18}$ is found to be singlet $\left(\mathrm{S}_{0}\right)$ for each tested functional. Analysis of the $\mathrm{C}_{18}$ structure in first triplet state $\left(\mathrm{T}_{1}\right)$ revealed that regardless of the applied functional the geometry of the $T_{1}$ corresponds to the polyynic structure. Our predictions are in good agreement with the experimental data for structurally similar polyynic chains. ${ }^{13,14}$

Structural changes observed in $T_{1}$ state compared to $S_{0}$ and a detailed analysis of HOMO and LUMO are given in Figures S2. We note that both HOMO and LUMO belong to the representations $\mathrm{E}_{4}{ }^{\prime}$ and $\mathrm{E}_{4}{ }^{\prime \prime}$, respectively. Experimental ${ }^{8}$ and theoretical ${ }^{7}$ studies confirmed that the structural/electronic characteristics of $\mathrm{C}_{18}$ do not change much by its adsorption on the $\mathrm{NaCl}$ surface and thus our gas-phase results appear to be applicable to the adsorbed system.
Keeping in mind the high electron affinity of $\mathrm{C}_{18}$ as well as reversible switching between its neutral and anion-radical forms, ${ }^{8}$ we consider photoinduced electron transfer (PET) processes in several van der Waals ( $\mathrm{vdW}$ ) complexes formed by $\mathrm{C}_{18}$ with typical donor and acceptor molecules widely applied in organic photovoltaics. The selected molecules exhibit both electron-donating and electron-accepting properties (Figure 2). As a typical electron-accepting units we selected fullerene $C_{60}$, perylenediimide (PDI) and 5,10-dithiophene substituted naphtho[1,2-c:5,6-c']bis([1,2,5]thiadiazole) (NTDA). These molecules are colored in blue in Figure 2. A typical electrondonating units are represented by zinc-porphyrin (ZnP), zincphthalocyanine (ZnPc), tetrathiafulvalene (TTF), 4,8dithiophene substituted benzo[1,2-b:4,5-b']dithiophene (BDT), and pentacene (PNTCN), they are colored in green.

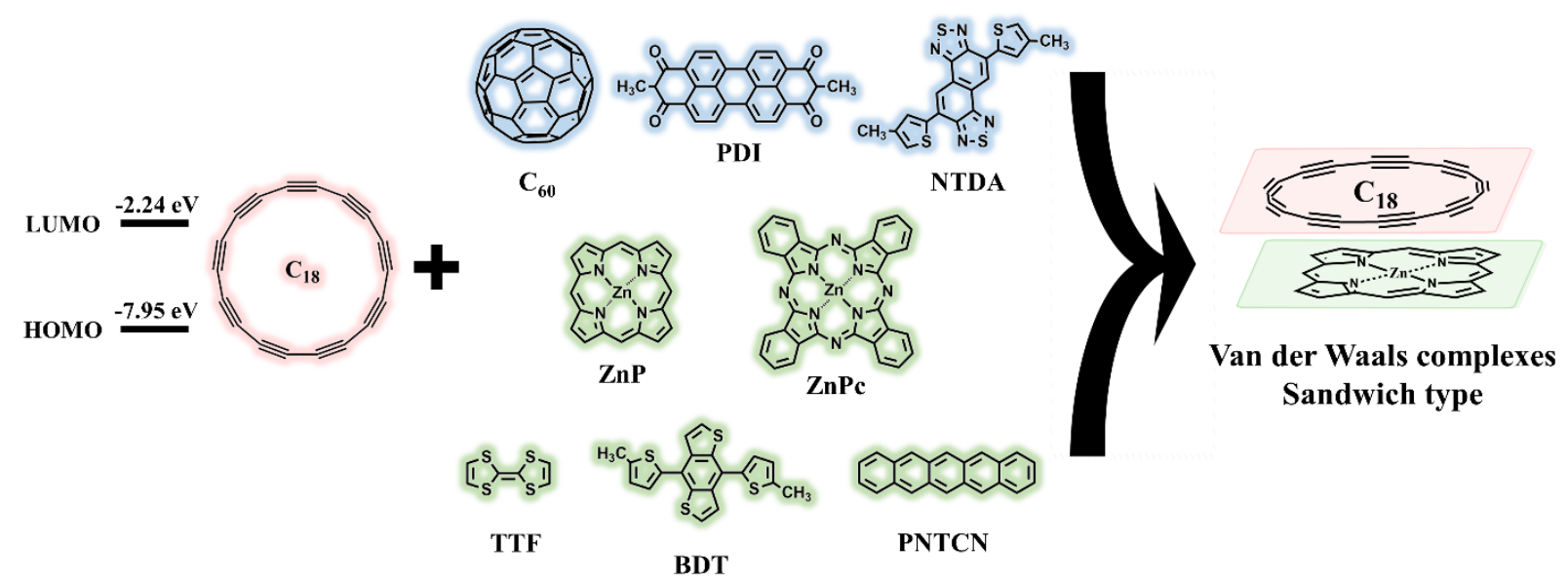

Figure 2. HOMO and LUMO energies of the $\mathrm{C}_{18}$ cluster and graphical representation of its partners in van der Waals complexes.

Geometries of the vdW complexes were optimized using CAMB3LYP-D3(zero-damping)/Def2-SVP. The obtained structures of the complexes are given in Figure S3, SI. The excited state calculations were performed using time-dependent DFT at the same level of theory (see full computational details in the SI). To characterize excited state properties, the studied complexes were divided into two fragments: $C_{18}$ moiety and its partner $\left(F_{x}\right.$ fragment). Exciton delocalization and charge transfer contributions were analyzed for the 80 lowest excited states of each complex. Three types of excited states were identified: locally excited states (LE) - exciton is mostly localized on a single fragment (charge separation value $<0.1 \mathrm{e}$ ); charge-separated (CS) states, where electron density is transferred between two fragments (charge separation $>0.8 \mathrm{e}$ ); and mixed states with significant contributions of LE and CS (charge separation is in between $0.1 \mathrm{e}$ and $0.8 \mathrm{e}$ ). Table 3 shows selected excited states properties of the studied vdW complexes. In each case, the lowest excited state is a LE located on the $\mathrm{C}_{18}$ fragment. The considered CS state corresponds to the electron transfer from $\mathrm{F}_{\mathrm{X}}$ to the $\mathrm{C}_{18}$ unit. This type of the CS state is the lowest-lying charge-separated state for all considered complexes (for details see Table S2). The non-adiabatic electron transfer rate, $\mathrm{k}_{\mathrm{ET}}$, was estimated using the classical Marcus equation ${ }^{15}$ (for details see the Supporting Information). It is important to note that the $\mathrm{C}_{18}$ cluster, in contrast to $\mathrm{C}_{60}$ and other fullerene electron acceptors, exhibits notable (about $0.4 \mathrm{eV}$ ) reorganization energy (Table S3, SI). As hown before, the reorganization energy is well estimated with the CAM-B3LYP method. The PET in the $\mathrm{C}_{18}$ complexes with electron-accepting units $\left(\mathrm{C}_{60}, \mathrm{PDI}\right.$, and NTDA) is characterized by notable positive Gibbs energies making this process highly unfavorable. In the case of $\mathrm{ZnP}$ and BDT partners, the electron transfer reaction exhibits nearly $0 \mathrm{eV}$ Gibbs energy, while in all other cases the Gibbs energies are quite negative. For all complexes of the $C_{18}$ with electrondonating partners, the PET occurs in normal Marcus regime $\left(\left|\Delta \mathrm{G}^{0}\right|<\lambda\right)$ on the nanosecond to picosecond time scales. For PNTCN, the limit of the normal Marcus regime is reached with $\Delta G^{0} \approx-\lambda$ and, consequently, this system has the highest predicted rate of ET.

Table 3. Interaction energies $\left(\Delta E_{\text {int }}, \mathrm{kcal} / \mathrm{mol}\right)$, singlet excitation energies $(\mathrm{E}, \mathrm{eV})$, oscillator strength (f), and degree of exciton localization $(\mathrm{X})$ on $\mathrm{C}_{18}$ fragment, extent of charge separation (CS, e), as well as electron transfer parameters - Gibbs energy $\left(\Delta G^{0}, e V\right)$, electronic coupling $(|V|, e V)$, reorganization energy $(\lambda, e V)$ and electron transfer rate $\left(k_{E T}, s^{-1}\right)$ for charge separation reactions of the studied vdW complexes. 


\begin{tabular}{|c|c|c|c|c|c|c|c|c|}
\hline & vdW complex $\left(F_{x}+C_{18}\right)$ & $\Delta \mathrm{E}_{\text {int }}$ for $\left(\mathrm{F}_{\mathrm{X}}+\mathrm{C}_{18}\right)^{\mathrm{a}}$ & Lowest LE $\left(\mathrm{C}_{18}\right)$ & $\mathrm{CS}\left(\mathrm{F}_{\mathrm{x}} \rightarrow \mathrm{C}_{18}\right)$ & $\Delta \mathrm{G}^{\mathrm{Ob}}$ & $|\mathrm{V}|$ & $\lambda$ & $\mathrm{k}_{\mathrm{ET}}$ \\
\hline & & & $E=2.571$ & $E=3.613$ & & & & \\
\hline \multirow[t]{3}{*}{1} & $\mathrm{C}_{60}+\mathrm{C}_{18}$ & -12.56 & $f<0.001$ & $f<0.001$ & 1.042 & 0.0108 & 0.509 & [n/a] \\
\hline & & & $X=0.99$ & $\mathrm{CS}=0.81$ & & & & \\
\hline & & & $E=2.583$ & $E=3.167$ & & & & \\
\hline \multirow[t]{3}{*}{2} & $\mathrm{PDI}+\mathrm{C}_{18}$ & -16.53 & $f<0.001$ & $f=0.003$ & 0.446 & 0.0292 & 0.555 & [n/a] \\
\hline & & & $X=0.98$ & $\mathrm{CS}=0.80$ & & & & \\
\hline & & & $E=2.588$ & $E=3.003$ & & & & \\
\hline \multirow[t]{3}{*}{3} & $\mathrm{NTDA}+\mathrm{C}_{18}$ & -17.21 & $f<0.001$ & $f=0.004$ & 0.415 & 0.0213 & 0.581 & [n/a] \\
\hline & & & $X=0.98$ & $\mathrm{CS}=0.81$ & & & & \\
\hline & & & $E=2.593$ & $E=2.185$ & & & & \\
\hline \multirow[t]{3}{*}{4} & $\mathrm{ZnPc}+\mathrm{C}_{18}$ & -20.25 & $f<0.001$ & $f=0.016$ & -0.408 & 0.0042 & 0.486 & $3.76 \cdot 10^{11}$ \\
\hline & & & $X=0.97$ & $C S=0.97$ & & & & \\
\hline & & & $E=2.581$ & $E=2.607$ & & & & \\
\hline \multirow[t]{3}{*}{5} & $\mathrm{ZnP}+\mathrm{C}_{18}$ & -16.38 & $f<0.001$ & $f<0.001$ & 0.026 & 0.0016 & 0.492 & $3.04 \cdot 10^{8}$ \\
\hline & & & $X=0.91$ & $\mathrm{CS}=0.86$ & & & & \\
\hline & & & $E=2.591$ & $E=1.991$ & & & & \\
\hline \multirow[t]{3}{*}{6} & $\mathrm{TTF}+\mathrm{C}_{18}$ & -11.93 & $f<0.001$ & $f=0.011$ & -0.600 & 0.0032 & 0.931 & $5.67 \cdot 10^{10}$ \\
\hline & & & $X=0.99$ & $C S=0.97$ & & & & \\
\hline & & & $E=2.569$ & $E=2.629$ & & & & \\
\hline \multirow[t]{3}{*}{7} & $\mathrm{BDT}+\mathrm{C}_{18}$ & -13.99 & $f<0.001$ & $f<0.001$ & 0.060 & 0.0209 & 0.657 & $4.47 \cdot 10^{9}$ \\
\hline & & & $X=0.90$ & $C S=0.96$ & & & & \\
\hline & & & $E=2.604$ & $E=2.135$ & & & & \\
\hline \multirow[t]{2}{*}{8} & PNTCN $+\mathrm{C}_{18}$ & -12.91 & $f<0.001$ & $f=0.020$ & -0.469 & 0.0117 & 0.489 & $3.26 \cdot 10^{12}$ \\
\hline & & & $X=0.98$ & $\mathrm{CS}=0.92$ & & & & \\
\hline
\end{tabular}

${ }^{\mathrm{a}} \Delta \mathrm{E}_{\mathrm{int}}=\mathrm{E}_{\mathrm{C} 18+\mathrm{Fx}}-\left(\mathrm{E}_{\mathrm{C} 18}+\mathrm{E}_{\mathrm{Fx}}\right) ;{ }^{\mathrm{b}}$ Gibbs energy difference between lowest LE and CS states.

In summary, we have demonstrated that the minimum energy structure for the $\mathrm{C}_{18}$ cluster predicted by the DFT method clearly depend on the amount of exact (HF) exchange in the particular DFT functional. Functionals with a high amount of HF exchange, such as M11 and M06-HF, give geometrical parameters to be close to the reference CCSD values. The low LUMO energy of $\mathrm{C}_{18}$ suggests that this molecule is a strong electron acceptor. We have studied several vdW complexes of the $C_{18}$ cluster with typical electron donor and acceptor molecules. The results show that the PET process is favorable only for complexes with such donors as ZnPc, TTF, and PNTCN where the CS state is the lowest-lying one and corresponds to the electron transfer from electron-donating unit to the $\mathrm{C}_{18}$ fragment. The PET occurs in normal Marcus regime on nanosecond to picosecond time scales. Thus, cyclo[18]carbon cluster is the smallest all-carbon electron-accepting unit reported so far.

We are grateful for financial support from the Spanish MINECO (Network RED2018-102815-T, project CTQ2017-85341-P, and Juan de la Cierva formación contracts FJCl-2016-29448 to A.J.S. and $\mathrm{FJCl}-2017-32757$ to O.A.S.), and the Catalan DIUE (2017SGR39, XRQTC, and ICREA Academia 2014 Award to M.S.).

\section{Conflicts of interest}

There are no conflicts to declare.

\section{Notes and references}

1 (a) F. Diederich, Y. Rubin, C. B. Knobler, R. L. Whetten, K. E. Schriver, K. N. Houk and Y. I. Li, Science, 1989, 245, 1088; (b) G. von Helden, N. G. Gotts and M. T. Bowers, Nature, 1993, 363, 60-63; (c) V. Parasuk, J. Almlof, M. W. Feyereisen, J. Am. Chem. Soc. 1991, 113, 1049-1050; (d) J. Hutter, H. P. Luethi, F. Diederich, J. Am. Chem. Soc. 1994, 116, 750-756.
2 (a) F. Diederich, Y. Rubin, Angew. Chem. Int. Ed. 1992, 31, 1101-1123; (b) F. Diederich, Nature 1994, 369, 199-207; (c) F. Diederich, M. Kivala, Adv. Mater. 2010, 22, 803-812.

3 (a) M. S. Islam, A. K. Ray, J. Phys. B: At., Mol. Opt. Phys. 1989, 22, 2071-2079; (b) C. Neiss, E. Trushin, A. Görling, ChemPhysChem 2014, 15, 2497-2502; (c) S. Sen, P. Seal, S. Chakrabarti, Phys. Rev. B 2006, 73, 245401; (d) T. Torelli, L. Mitas, Phys. Rev. Lett. 2000, 85, 1702-1705.

4 (a) R. Hoffmann, Tetrahedron 1966, 22, 521-538; b) P. W. Fowler, N. Mizoguchi, D. E. Bean, R. W. A. Havenith, Chem. Eur. J. 2009, 15, 6964-6972.

5 D. A. Plattner, K. N. Houk, J. Am. Chem. Soc. 1995, 117, 44054406.

6 S. Arulmozhiraja, T. Ohno, J. Chem. Phys. 2008, 128, 114301.

7 G. V. Baryshnikov, R. R. Valiev, A. V. Kuklin, D. Sundholm, and H. Ågren, J. Phys. Chem. Lett. 2019, 10, 6701-6705

8 K. Kaiser, L. M. Scriven, F. Schulz, P. Gawel, L. Gross, H. L. Anderson, Science 2019, 365, 1299-1301.

9 (a) M. G. Medvedev, I. S. Bushmarinov, J. Sun, J. P. Perdew, K. A. Lyssenko, Science 2017, 355, 49; (b) N. Mardirossian, M. Head-Gordon, Mol. Phys. 2017, 115, 2315-2372.

10 The formula for the B3LYP energy is: $E_{X C}^{B 3 L Y P}=E_{X}^{L D A}+\alpha_{0}\left(E_{X}^{H F}-E_{X}^{L D A}\right)+\alpha_{X}\left(E_{X}^{G G A}-E_{X}^{L D A}\right)+E_{C}^{L D A}+\alpha_{C}\left(E_{C}^{G G A}-E_{C}^{L D A}\right)$ In this work the $\alpha_{0}$ coefficient varies from 0.05 to 1.00 . $\alpha_{x}=1-$ $\alpha_{0}$; while $\alpha_{c}=0.81$.

11 List of used XC functionals with corresponding references are listed in Table S1.

12 (a) B. Braïda, P. C. Hiberty, A. Savin, J. Phys. Chem. A 1998, 102, 7872-7877; (b) J. Poater, M. Solà, A. Rimola, L. Rodríguez-Santiago, M. Sodupe, J. Phys. Chem. A 2004, 108, 6072-6078.

13 (a) T. Pino, H. Ding, F. Güthe, J. P. Maier, J. Chem. Phys. 2001, 114, 2208-2212; (b) M. Mahbubul Haque, L. Yin, A. R. T. Nugraha, R. Saito, Carbon 2011, 49, 3340-3345.

14 H. Yoneda, H. Hiura, H. Takahashi, J. Mol. Struct. 1993, 301, 47-56.

15 R. A. Marcus, N. Sutin, Biochim. Biophys. Acta, Rev. Bioenerg. $1985,811,265-322$ 